\title{
Gordofobia: Una deuda en el campo de la psicología
}

\author{
Fatphobia: A debt in the field of psychology \\ Gordofobia: uma dívida no campo da psicologia
}

Ana María Gallardo*

\section{RESUMEN}

La gordofobia se puede entender como las actitudes, estereoti-

Palabras clave: pos sociales negativos y prejuicios hacia las personas con sobrepeso (Harris et al., 1990), que pueden ser acompañados de actos de violencia (Noronha \& Deufel, 2014), barreras ambientales (Friedman et al., 2008) o barreras sociales (De Domingo y López, 2014). De este modo, la "gordofobia" compone un sistema de opresión que pone al sujeto en una situación de desventaja, injusticia y exclusión, que se reproduce de forma sistemática y estructural (Bastos, 2019).

El presente artículo de reflexión tiene como objetivo plantear el estudio de la gordofobia como una tarea pendiente dentro de la psicología, y cuestionar los paradigmas desde los cuales se entiende el cuerpo.

\section{SUMMARY}

Fatphobia can be understood as attitudes, negative social stereotypes, and prejudices towards overweight people (Harris et al., 1990), which can be accompanied by acts of violence (Noronha \& Deufel, 2014), environmental barriers (Friedman et al., 2008) or social barriers (De Domingo \& Lopez, 2014). Thus, "fatphobia" composes a system of oppression that puts the subject in a situation of disadvantage, injustice, and exclusion, which is reproduced systematically and structurally (Bastos, 2019). The present gordofobia, estereotipo, estigma, género, equipos de salud y educación, enfoque interseccional

Key words: fatphobia, stereotyping, stigma, gender, health, and education teams, intersectional approach article aims to propose the study of fatphobia as a pending task within psychology. Also, to question the paradigms from which the body is understood.

\footnotetext{
* Chilena. Centro de Estudios y Atención Clínica con Enfoque de Cuerpo y Género.
} amgallar@gmail.com 


\section{RESUMO}

A gordofobia pode ser entendida como as atitudes, estereótipos

Palavras-chave: sociais negativos e preconceitos em relação às pessoas com sobrepeso (Harris et al., 1990), que podem ser acompanhados de atos de violência (Noronha e Deufel, 2014), barreiras ambientais (Friedman et al., 2008) ou barreiras sociais (De Domingo e López, 2014). Deste modo, a "gordofobia" compõe um sistema de opressão que coloca o sujeito em uma situação de desvantagem, gordofobia, estereótipo, estigma, gênero, equipes de saúde e educação, enfoque interseccional injustiça e exclusão, que se reproduz de forma sistemática e estrutural (Bastos, 2019).

O presente artigo de reflexão tem como objetivos propor o estudo da gordofobia como uma tarefa pendente dentro da psicologia e questionar os paradigmas desde os quais entende-se o corpo. 


\section{Obesidad}

El sobrepeso y obesidad son definidos como una acumulación excesiva de grasa corporal que es perjudicial para la salud. En el caso de los adultos, la Organización Mundial de la Salud (OMS, 2020) define el sobrepeso a partir de un Índice de Masa Corporal (IMC) $\geq 25 \mathrm{~kg} / \mathrm{m}^{2}$, y la obesidad a partir de un IMC $\geq 30,0 \mathrm{~kg} / \mathrm{m}^{2}$.

Se estima que, desde 1975, la obesidad se ha casi triplicado en todo el mundo. Es así como, para 2016, más de 1.900 millones de adultos de 18 o más años tenían sobrepeso, de los cuales más de 650 millones eran obesos (OMS, 2020).

En el caso específico de Chile, el IMC promedio de la población adulta se ha incrementado drásticamente en las últimas cuatro décadas, pasando de 24,2 a $28,1 \mathrm{~kg} / \mathrm{m}^{2}$ y de 24,8 a $29,4 \mathrm{~kg} / \mathrm{m}^{2}$ en hombres y mujeres, respectivamente (Petermann et al., 2020) y ocupando actualmente el segundo lugar en los países con mayor prevalencia de obesidad, con un 34,4\% de su población mayor de 15 años presentándola (Martínez et al., 2020).

El sobrepeso y la obesidad tienen consecuencias importantes en la vida y salud de las personas, elevando el riesgo en el desarrollo de enfermedades cardiovasculares, diabetes, trastornos del aparato motor y algunos tipos de cáncer (por ejemplo, endometrio, mama, ovarios, próstata, hígado, vesícula biliar, riñones y colon) (OMS, 2020).

Sin embargo, las consecuencias en la salud física no son las únicas importantes de destacar, ya que se ha vinculado el sobrepeso y obesidad a un deterioro en la salud mental. Por ejemplo, la American Psychological Association señala que, a menudo, la obesidad está acompañada de depresión grave y pensamientos de suicidio.

A la vez, la depresión, el estrés o eventos repentinos y emocionalmente agotadores, pueden generar cambios en los hábitos de alimentación y ejercicios que, con el tiempo, se configuran como hábitos difíciles de cambiar (APA, 2020). De este modo, más que considerar que son cuadros comórbidos, la evidencia señala que ambos cuadros se influyen mutualmente en la presentación, trayectoria y resultado (Alonso y Olivos, 2020).

Si bien existe evidencia que señala que tanto la depresión como la obesidad son trastornos de la desregulación del sistema de estrés 
y asociadas a una condición inflamatoria crónica (Ibíd.), en este artículo se reflexionará acerca de los efectos del estigma social en las personas, puesto que se ha evidenciado el efecto de la discriminación en la salud en general y en el bienestar social y emocional (Saavedra y Robles, 2011).

De este modo, el primer objetivo del presente artículo será definir el concepto de "gordofobia", entender los pilares en que se sustenta este tipo de discriminación, proponer una mirada interseccional de la gordofobia y, finalmente, reflexionar por qué el estudio de la gordofobia es una deuda en el campo de la psicología. Esta discusión permite iniciar el estudio de la internalización del estigma en las personas, su efecto en su autoestima y autoimagen, y la relación con los equipos de salud.

\section{Gordofobia}

La gordofobia se puede entender como el miedo a la gordura (Robinson, Bacon \& O'Reilly, 1993), o como las actitudes, estereotipos sociales negativos y prejuicios hacia las personas con sobrepeso (Harris, Waschull \& Walters, 1990; Robinson et al., 1993), acompañados de actos de violencia física, verbal, moral, psíquica (Noronha \& Deufel, 2014), barreras ambientales a las que se enfrentan a diario, como los espacios pequeños (Friedman, Ashmore \& Applegate, 2008) o barreras sociales, como es la discriminación en espacios laborales, puesto que las personas con sobrepeso y obesidad tienen mayor desempleo (De Domingo y López, 2014)

Desde la literatura científica, el concepto más utilizado para referir a la discriminación asociada a las personas con sobrepeso es el de "sesgo por peso" (Weight Bias), el cual se define como la inclinación a realizar juicios no razonables con base en el peso de la persona (Washington, 2011).

El sesgo por peso ha sido bastante estudiado, encontrándose 26.231 artículos publicados en PubMed entre 1960 y 2020, con un peak el año 2018, con 1.913 artículos.

Sin embargo, el presente artículo direcciona la reflexión hacia la incorporación del concepto "gordofobia", puesto que dicho concepto alude a un sistema de opresión, que pone al sujeto en una situación de desventaja, injusticia y exclusión, y que se reproduce de forma siste- 
mática y estructural (Bastos, 2019), ajustándose con mayor precisión a lo que viven las personas con sobrepeso y obesidad.

No obstante, la base de datos previamente citada solo reporta 122 artículos publicados entre 1982 y 2020, con un peak de ocho artículos publicados en 2013. De este modo, se observa la necesidad de continuar desarrollando el concepto de "gordofobia" y ampliar la investigación acerca de los efectos que tiene en quienes reciben la discriminación.

De este modo, se abordará el concepto de "gordofobia" desde un enfoque de género, estética, discursos asociados a la salud física y el temor que implica ser parte de un grupo que vive constantemente bajo la lupa del escrutinio social.

\section{Una mirada psicosocial: estereotipo y estigma de la persona gorda}

Los estereotipos se refieren a las creencias populares sobre los atributos que caracterizan a un grupo social (Mackie, 1973). Considerando los estudios previamente citados, se comprende que la sociedad ha formado un estereotipo de persona gorda, que es indisciplinada, inactiva, poco atractiva, con falta de autocontrol y que, además, sufriría de problemas emocionales y psicológicos, tales como baja autoestima, inseguridad y depresión (Robinson et al., 1993).

Un estudio llevado a cabo por Gallardo y Franco (2020) muestra cómo un grupo de mujeres asocia la palabra "gorda" a una persona con bajo nivel de bienestar emocional y salud mental, malestar y problemas físicos y baja valoración social. De este modo, se puede observar que, al menos para el grupo encuestado, la palabra "gorda" no solo es una talla o un peso, sino que un concepto con un profundo significado social asociado.

Definir un estereotipo asociado a un grupo de personas, implica adentrarse en el estigma social vinculado a la imagen construida acerca de dicho grupo. Específicamente, un estigma se refiere a una "condición, atributo, rasgo o comportamiento que hace que su portador genere una respuesta negativa y sea visto como «culturalmente inaceptable» o inferior" (Flores, Medina, Robles, 2011, p. 334), y que además justifica la exclusión social de quienes los poseen (Pla, Adam y 
Bernabeu, 2013). Dichas respuestas pueden ser verbales - tales como burlas, sobrenombres, insultos-; físicas - manifestaciones de violencia-, y otras barreras y obstáculos que se traducen en discriminación individual o colectiva (De Domingo y López, 2014)

De este modo, se comprende que cada sociedad define ciertas pautas para que las personas sean categorizadas como "normales" o "desviantes" (Goffman, 1963, en Navajas, 2017). Tal como se ha revisado, el sobrepeso se desvía tanto de la norma estética como del ideal de salud. De este modo, el cuerpo hipervisible, diferente, se vuelve fuente de agresión y estigmatización social (Energici et al., 2017; Navajas, 2017), amparada por estos modelos de cuerpo ideal, belleza hegemónica y la falsa creencia de que los comentarios emitidos hacia dichos cuerpos deberían tener una consecuencia positiva en el cambio de hábitos alimentarios en la persona (Jackson, 2016).

En este caso, dicho estigma se reproduce en distintos ámbitos de la vida de las personas, incluyendo la familia, el trabajo, la escuela, e incluso en la atención médica (Friedman et al., 2008; OAC, 2018). Al ser el sobrepeso algo visible a los ojos de los otros, las personas deben convivir con la constante evaluación de terceros. De este modo, las personas no solo conviven con la constante devaluación, sino que además desarrollan el temor a pertenecer a este grupo estigmatizado (Palmeira, Cunha \& Pinto-Gouveia, 2018).

Por esto, Gallardo y Franco (2020) proponen que, dentro del campo de la gordofobia, también se debería incorporar la noción de "estigma internalizado de la gordofobia". Específicamente, el concepto de "estigma internalizado" describe el hecho de que las personas comienzan a internalizar los estereotipos que se les asignan (Muñoz, Sanz y Pérez, 2011). A diferencia del estigma estructural y social, el internalizado afecta aspectos subjetivos, tales como la esperanza, autoestima, empoderamiento, autoeficacia (Garrido, 2011; Wright, Gronfein, \& Owens, 2000). En el caso de las personas con sobrepeso, ellas tienden a internalizar los estereotipos negativos y a culparse a sí mismas, lo que genera un desmedro en su bienestar emocional (OAC, 2018).

Estudios asociados a la actitud negativa, prejuicios y discriminación hacia personas con obesidad, han mostrado que no solo existen dichos tratos y discursos hostiles en la población general, sino que 
también son cometidos por profesionales de la salud (Friedman et al., 2008), adulta y pediátrica (Puhl \& Latner, 2007), y educadores (Nutter et al., 2019), por lo que afectan a la persona en el día a día, durante toda su vida.

A continuación se revisarán algunos de los pilares que han sustentado los estereotipos asociados al cuerpo gordo en la sociedad actual, y que, a la vez, han perpetuado los sistemas de discriminación a personas de tallas diversas.

\section{Discusión desde la perspectiva de género y los cánones de belleza}

Se ha señalado que, desde temprana edad, las mujeres están expuestas a más comentarios públicos acerca de sus cuerpos que los hombres (Fredrickson, Roberts, Noll, Quinn \& Twenge, 1998; González, Valdez, Serrano, 2003). Por ejemplo, en un estudio llevado a cabo por Gallardo (2019) en el que participaron 299 mujeres, 72,6\% de las participantes señaló haber recibido comentarios percibidos como molestos o hirientes relacionado con el tamaño (peso) de sus cuerpos.

En esta línea, Fredrickson y colegas $(1998,2005)$ plantean la teoría de la autocosificación, la cual explica que, dado que la mujer ha sido enseñada a sostener un monitoreo vigilante hacia su cuerpo, desarrolla mayor autoconciencia respecto de este.

En este sentido, ya que en la sociedad actual existe un ideal de belleza basado en la delgadez (Matacin \& Simone, 2019; Suárez, 2017), la autocosificación en la mujer se relaciona con mayor vergüenza hacia su propio cuerpo, ansiedad, menor conciencia hacia sus señales del cuerpo y restricciones alimentarias entre problemas relacionados con la salud mental.

Junto con el reforzamiento del ideal de cuerpo que se exhibe en los medios (Gapinski, Brownell \& LaFrance, 2003), la mujer está más propensa a desarrollar una noción del cuerpo instrumental y modificable, y a la utilización de cirugías cosméticas para lograr el ideal de belleza (Muñiz, 2014; Pla, Adam y Bernabeu, 2013).

Desde este punto de vista, el cuerpo de la mujer no solo es su peso y estatura, sino que los criterios estéticos establecidos socialmente desde los que se evalúa (Cardona, 2015). Se postula entonces que existirían 
más actitudes negativas, discriminación o gordofobia hacia el cuerpo femenino gordo, que hacia el cuerpo masculino gordo (Crandall \& Biernat, 1990; Hayran, Akan, Ozkan \& Kocaoglu, 2013; Suárez, 2017).

\section{Discusiones desde el ámbito de la salud}

Respecto de la salud de las personas, existe la creencia de que la obesidad es resultado de una falta de autocontrol personal y de malas elecciones individuales, lo que ha llevado a adoptar estilos de vida poco saludables (Navajas, 2017). Desde esta mirada, la obesidad es percibida como un problema individual, en vez de comprenderlo desde una mirada ambiental, social y política (Christiansen, 2018; Hayran et al., 2013).

Del mismo modo, se ha cuestionado el tono alarmista que implica el discurso de la "epidemia de obesidad", en la medida que utilizan conceptos tales como "la guerra contra la obesidad", con un foco puesto en el gasto social en salud más que en el bienestar de las personas (Campos, Saguy, Ernsberger, Oliver \& Gaesser, 2006; Navajas, 2017). Estos discursos, utilizados tanto en los medios de comunicación como en políticas públicas, han generado una demonización de la gordura y una idealización de la delgadez (Alberga, Russel-Mayhew, Von Ranson \& McLaren, 2016), implicando un cuestionamiento moral hacia las personas con sobrepeso (Campos et al., 2006).

La relevancia de poner este tema como eje de discusión, es que existe evidencia de que el personal de los equipos de salud tiende a tener sesgos hacia las personas con sobrepeso (Puhl \& Heuer, 2010), reportando actitudes negativas hacia los pacientes obesos (Thuan \& Avignon, 2005) e, incluso, reportando bajar la calidad de la atención médica cuando los pacientes tienen sobrepeso (Washington, 2011).

Dichos sesgos son percibidos por los pacientes. Por ejemplo, 31,8\% de las mujeres que participaron en un estudio relacionado con la actitud hacia el cuerpo de la mujer, informó haber recibido comentarios hacia su cuerpo (peso) percibidos como incómodos por parte de profesionales de la salud a causa del sobrepeso (Gallardo, 2019). Esto sucede porque existe una falsa creencia, respecto de que este tipo de actitudes podrían promover conductas de vida saludable en las personas (Puhl \& Brownell, 2003; Puhl \& Heuer, 2010). 
Es importante señalar que el foco del presente artículo no es negar las consecuencias asociadas al sobrepeso y la obesidad, sino que visibilizar cómo los discursos biomédicos han contribuido a la estigmatización de la obesidad, y cómo esto, a la vez, aleja a las personas que requieren apoyo para bajar de peso (Puhl \& Heuer, 2010). Es importante recalcar que, si se habla de potenciar la salud, las personas se motivan más por mensajes que se focalizan en comportamientos saludables y que promueven la aceptación de cuerpo (Alberga et al., 2016; Harriger \& Thomson, 2012), en vez de aquellos que se basan en el peso corporal (Alberga et al., 2016).

\section{Discusiones desde el ámbito de la educación}

Se ha discutido acerca de los efectos de la obesidad en la salud de niños, niñas y adolescentes, pero poco se ha estudiado acerca de las consecuencias psicológicas que implica el sobrepeso, tales como baja autoestima, depresión, insatisfacción corporal y trastornos de la conducta alimentaria (Harriger \& Thomson, 2013). Por ejemplo, un estudio llevado a cabo en Chile con escolares entre los 11 y 13 años, señala que niños con sobrepeso y obesidad tenderían a tener peor autoestima y mayor sensación de infelicidad (Delgado, Caamaño, Martinez, Jerez, Carter, García \& Latorre, 2018).

El motivo del malestar que presentan los escolares con sobrepeso es que suelen ser víctimas de agresiones verbales, físicas y sociales (exclusión, y ser centro de rumores) desde la etapa preescolar hasta la vida adolescente. Dichas discriminaciones son efectuadas por parte de pares, profesores y otros profesionales de la educación, y se basan en las creencias erróneas que tienen acerca de individuos obesos (Nutter et al., 2019).

Por parte de los profesionales de la educación, el objetivo de "combatir" la obesidad y las acciones para mejorar la salud de los estudiantes, algunas veces adopta estrategias potencialmente peligrosas (Nutter et al., 2019; Russell \& Grace, 2015). Por ejemplo, mensajes focalizados en la incomodidad del sobrepeso podrían contribuir en niños y adolescentes a la sensación de sentirse inadecuados (O’Dea, 2015). Del mismo modo, prácticas como medir y pesar a los alumnos en el ramo de educación física podrían percibirse como una forma de humillar y disciplinar a las personas de cuerpos gordos (Sykes \& McPhail, 2008). 
Por otra parte, el sesgo por peso también influye en la percepción que tienen los profesores respecto de alumnos con obesidad (Nutter et al., 2019). Por ejemplo, algunos estudios han señalado que los docentes sostendrían que alumnos con obesidad tendrían más desafíos académicos (Kenney, Redman, Criss, Sonneville \& Austin, 2017), y que incluso niñas con obesidad rendirían menos en lenguaje, mientras que niños con obesidad tendrían menos habilidades en matemáticas (Kenney, Gortmaker, Davison \& Austin, 2015).

De este modo, tal como se señala en el apartado anterior, el foco del artículo no es negar las consecuencias que tiene el sobrepeso y la obesidad en la salud de los escolares, sino que alertar que existen sesgos negativos hacia niños, niñas, y adolescentes con sobrepeso en el sistema educativo, y que la discriminación por peso es un tema serio que requiere mayor atención (Harriger \& Thomson, 2013) y entrenamiento específico para combatir sesgos, creencias y actitudes gordofóbicas en los equipos docentes (O'Dea, 2005).

\section{Interseccionalidad}

Comprendiendo que el estigma de la gordura no puede analizarse solo desde la vertiente del género, cánones de belleza, salud o educación, es necesario adoptar una mirada interseccional del estigma del peso.

El concepto de "interseccionalidad" es acuñado por Kimberlée Crenshaw (1989) para analizar las múltiples y simultáneas estructuras de opresión que interactúan para dar forma a complejas discriminaciones de mujeres negras en Estados Unidos. En este sentido, Crenshaw explica que no se trata de una suma de desigualdades, ya que "la experiencia interseccional es más grande que la suma de racismos y sexismos" (p. 4).

En esta línea, un enfoque interseccional critica la identidad basada en un sujeto supuestamente universal - masculino, occidental/blanco, heterosexual, adulto y burgués - como referente (Cubillos, 2015) y propone múltiples categorías sociales interconectadas (por ejemplo, etnia, género) para analizar cómo dichas categorías afectan la salud (Himmelstein, Puhl \& Quinn, 2017).

En este sentido, es importante problematizar que existe poca literatura relacionada con el estigma del peso en el campo de la salud y 
ciencias sociales, y menos aun considerando una mirada interseccional. Esto es relevante puesto que, en el caso de la obesidad, los juicios no solo se dirigen al tamaño del cuerpo, sino que se juzga sobre la base de discriminaciones anteriores, por ejemplo, por género, etnia, edad y/o nivel socioeconómico (Energici, Acosta, Borquez y Huaiquimilla, 2017; Hayran et al., 2013).

En cuando a la raza y la etnia, Strings (2019) afirma que la preferencia por el cuerpo delgado y la fobia al cuerpo gordo ha sido una forma de moldear y legitimar las jerarquías de clase con base en el sexo y la raza. Así, la autora realiza una revisión histórica desde los tiempos de la esclavitud negra, y sostiene que los discursos negativos tienen su raíz en los procesos de diferenciación por raza dentro de las dinámicas de poder.

De este modo, la existencia de un tipo racial europeo idealizado se ha impuesto como una aspiración para las mujeres de tez oscura y mujeres de países no occidentales, indígenas, mestizas, mulatas y asiáticas. Es así como la "raza" —inmutable desde la biología - también ha sido un factor de homogeneización de belleza, tan antigua como la colonización en distintos países (Muñiz, 2014).

Considerando el factor género y etnia explicados previamente, Himmelstein y colegas (2017) estudiaron los efectos del estigma del peso en mujeres de distintos orígenes étnicos, e informan que las mujeres hispanas son más propensas a desarrollar trastornos alimentarios y conductas alimentarias de riesgo para contrarrestar los efectos del estigma del peso que mujeres provenientes de otras etnias.

Otro cruce relevante es la relación estrecha en las actitudes negativas hacia las personas obesas y las personas de bajo nivel socioeconómico, puesto que en la base existe la idea que ambos grupos son flojos y que carecen de autocontrol (Campos et al., 2006; Crandall \& Biernat, 1990). En palabras de Energici y colegas (2017, p. 8): “La exclusión por el tamaño del cuerpo puede ser una nueva forma de clasismo, donde el cuerpo marca o materializa la pereza y la falta de autocontrol".

También es importante mencionar que los escasos estudios llevados a cabo acerca de la gordofobia y el estigma del cuerpo provienen de culturas occidentales, y no consideran necesariamente las realidades locales en cuanto a raza y colonialismos, clases, género y otras va- 
riables (Pausé, 2014), y cómo afecta a las personas de acuerdo con las categorías de discriminación que se suman al estigma de la gordura.

\section{Por qué es un tema relevante en la investigación en psicología}

Tal como se ha discutido durante el presente artículo, las consecuencias del sobrepeso y obesidad no se limitan a los síntomas y a los signos sanitarios, sino que transcienden al campo de las ciencias sociales (De Domingo \& López, 2014), teniendo consecuencias importantes en la vida de las personas, como la vergüenza y autodesprecio por el propio cuerpo (Plá, Adam y Bernabeu, 2013).

Considerando los aspectos analizados, se entiende que la autoaceptación y el bienestar psicológico no se determinan necesariamente por el peso corporal de las personas, sino que por las consecuencias derivadas de las experiencias sociales de discriminación y exclusión (Magallares, 2014)

Uno de los motivos por los que se sigue perpetuando el estigma, son los valores culturales que se asocian a la delgadez, y por la falsa creencia de que realizar una crítica o promover culpa hacia el cuerpo de la persona podría generar cambios en su conducta (Washington, 2011). Sin embargo, como profesionales de la salud mental debemos alertar acerca del efecto contrario que tienen dichas críticas: aumento de peso, desórdenes alimentarios, abandono de dietas y evitación de actividades físicas, desmejoran el bienestar físico y mental, además de disminuir la percepción de autoeficacia en torno a estilos de vida saludables y evitación de consultas médicas para mejorar la salud (Friedman et al., 2008; Himmelstein et al., 2017; Jackson, 2016; O’Dea, 2005).

Lamentablemente, a pesar de las consecuencias negativas en la vida de las personas, pareciera que no existe una evolución en la erradicación del estigma social hacia las personas con sobrepeso en las últimas décadas, sino que, al contrario, la cultura de la dieta la hace más visible. Tal como expresa Puhl y colegas (2015, p. 9): “Los sesgos hacia el peso son socialmente aceptables, raramente desafiados, y las sanciones y prohibiciones hacia prejuicios o discriminaciones son inexistentes". 
Debido a las secuelas emocionales que implica ser un grupo maltratado por la sociedad y por la institución (Navajas, 2017), hablar de discriminación hacia el cuerpo gordo es una suerte de reivindicación, e incluso una cuestión de justicia social (De Domingo \& López, 2014).

Para esto, es necesario que realicemos activismos dentro de nuestras áreas del saber y reflexionemos acerca de la propia gordofobia internalizada y normalizada. De este modo, este artículo es un llamado a continuar abordando la gordofobia en el campo de la investigación, e incorporarla a la reflexión en nuestra praxis clínica.

\section{Bibliografía}

Alberga, A., Russel-Mayhew, S., Von Ranson, K. \& McLaren, L. (2016). Weight bias: a call to action. Journal of Eating Disorders, 4(34), 1-6. DOI 10.1186/s40337-016-0112-4

Alonso, R. y Olivos, C. (2020). La relación entre la obesidad y estados depresivos. Revista Médica Clínica Las Condes, 31(2), 130138. DOI: https://doi.org/10.1016/j.rmclc.2020.02.004

APA (2 de agosto de 2020). Salud mental/corporal: La obesidad. Centro de Apoyo. https://www.apa.org/centrodeapoyo/obesidad.

Bastos, M. (2019). Os corpos gordos merecem ser vividos. Revista Estudos Feministas, 27(1), 1-6. DOI: 10.1590/1806-95842019v27n156453

Campos, P., Saguy, A., Ernsberger, P., Oliver, E. \& Gaesser, G. (2006). The epidemiology of overweight and obesity: public health crisis or moral panic? International Journal of epidemiology, 35(1), 55-60. DOI: 10.1093/ije/dyi254

Cardona, J. (2015) Cánones de belleza: La alienación femenina. Revista de Filosofía, (16), 26-30.

Christiansen, M. (2018). "Homo Caloricus": La construcción epistemológica del estigma lipofóbico en los discursos mediáticos de salud pública. Salud Colectiva, 14(3), 623-637. DOI: 10.18294/sc.2018.1602doi: 10.18294/sc.2018.1602

Crandall C, \& Biernat M. (1990). The ideology of anti-fat attitudes. Journal of Applied Social Psychology, (20), 227-243. https://doi. org/10.1111/j.1559-1816.1990.tb00408.x

Crenshaw, K. (1989). Demarginalizing the intersection of race and sex: A black feminist critique of antidiscrimination doctrine, fe- 
minist theory and antiracist politics. University of Chicago Legal Forum, (140), 139-167.

Cubillos, J. (2015). La importancia de la interseccionalidad para la investigación feminista. Oxímora, Revista Internacional de Ética y Política, (7), 119-137.

Delgado, P., Caamaño, F., Martinez, C., Jerez, D., Carter, B., García, F. y Latorre, P. (2018). La obesidad infantil y su asociación con el sentimiento de infelicidad y bajos niveles de autoestima en niños de centros educativos públicos. Nutrición Hospitalaria, (35), 533-537. DOI: http://dx.doi.org/10.20960/nh.1424

De Domingo, M. y López, G. (2014). La estigmatización social de la obesidad. Cuadernos de Bioética, 24(2), 273-284.

Energici, M. A., Acosta, E. y Borquez, F. y Huaiquimilla, M. (2017). Gordura, discriminación y clasismo: un Estudio en Jóvenes de Santiago de Chile. Psicologia \& Sociedade, (29), 1-10. DOI: https://doi.org/10.1590/1807-0310/2017v29164178

Flores, S., Medina, R. y Robles, R. (2011). Estudio de traducción al español y evaluación psicométrica de una escala para medir el estigma internalizado en pacientes con trastornos mentales graves. Salud Mental, (34), 333-339.

Friedman K., Ashmore J. \& Applegate K. (2008). Recent experiences of weight-based stigmatization in a weight loss surgey population: Psychological and behavioral correlates. Obesity, 16(2), 69-74. DOI: 10.1038/oby.2008.457

Fredrickson, B. L. \& Harrison, K. (2005). Throwing like a girl: Self-objectification predicts adolescent girls' motor performance. Journal of Sport and Social Issues, (29), 79-101.

Fredrickson B. L., Roberts T. A., Noll S. M., Quinn D. M. \& Twenge J. M. (1998). That swimsuit becomes you: sex differences in selfobjectification, restrained eating, and math performance. Journal Pers. Soc. Psychol, (75), 269-284. DOI: 10.1037/00223514.75.1.269

Gallardo, AM (2019). Cuestión de peso: Estudio exploratorio y comparativo de la actitud hacia el peso de las mujeres en relaciones cotidianas y profesionales de la salud en una muestra de mujeres chilenas. Summa Psicológica UST, 17(2), 195-203. DOI: 10.18774/0719-448.x2020.17.461

Gapinski, K. D., Brownell, K. D. \& LaFrance, M. (2003). Body objectification and "fat talk": Effects on emotion, motiva- 
tion, and cognitive performance. Sex Roles, (48), 377-378. DOI:10.1023/A:1023516209973

Garrido, M. (2011). La rehabilitación psicosocial en los trastornos mentales graves. Anuario de Psicología Clínica y de la Salud, (7), 2-5.

González, N., Valdez, J. y Serrano, J. (2003). Autoestima en jóvenes universitarios. Ciencia Ergo Sum, 10(2), 172-176.

Harriger, J. \& Thompson, K. (2012). Psychological consequences of obesity: Weight bias and body image in overweight and obese youth Jennifer. International Review of Psychiatry, 24(3), 247-253. DOI: 10.3109/09540261.2012.678817

Harris, M. B., Waschull, S., \& Walters, L. (1990). Feeling fat: Motivations, knowledge, and attitudes of overweight women and men. Psychological Reports, 67(3), 1191-1202. DOI: https:// doi.org/10.2466/PR0.67.8.1191-1202

Hayran, O., Akan, H., Ozkan, A. D. \& Kocaoglu, B (2013). Fat phobia of university students: attitudes toward obesity. Journal of Allied Health, (42), 147-150.

Himmelstein, M., Puhl, R. \& Quinn, D. (2017). Intersectionality: An Understudied Framework for Addressing Weight Stigma. American Journal of Preventive Medicine, 53(4), 421-431.

Kenney, E., Redman M., Criss, S., Sonneville K. \& Austin, S. (2017). Are K-12 school environments harming students with obesity? A qualitative study of classroom teachers. Eat Weight Disorder, (22), 141-152. DOI: https://doi.org/10.1007/s40519-016-0268-6

Kenney E., Gortmaker S., Davison, K. \& Austin SB. (2015) The academic penalty for gaining weight: a longitudinal, change-in-change analysis of BMI and perceived academic ability in middle school students. Epidemiology and Population Health, (9), 1408-1413. DOI: https://doi.org/10.1038/ijo. 2015.88

Mackie, M. (1973). Arriving at Truth by Definition: Case of Stereotype Innacuracy. Social Problems, (20), 431-447.

Magallares, A. (2014). Prejuicios, discriminación y calidad de vida en obesidad mórbida. En M.A. Rubio, M. D. Ballesteros-Pomar, Sánchez-Pernaute y Torres-García (Eds.), Manual de obesidad mórbida (pp. 79-84). Madrid: Panamericana.

Matacin, M. \& Simone, M. (2019) Advocating for Fat Activism in a Therapeutic Context. Women Ther, 42(1), 200-215. DOI:10.1080/ 02703149.2018.1524071. 
Martínez, M., Petermann, F., Villagrán, M., Ulloa, N., Nazar, G., Troncoso, C., Garrido, A., Mardones, L., Lanuza, F., Leiva, AM., Lasserre, N., Martorell, M. \& Celis, C. (2020). From a global view to the Chilean context: which factors have influenced the development of obesity in Chile? (Chapter 2). Revista chilena de nutrición, 47(2), 307-316. DOI: https://dx.doi. org/10.4067/S0717-75182020000200307

Muñoz, M., Sanz, M. y Perez, E. (2011). Estado del conocimiento sobre el estigma internalizado desde el modelo socio-cognitivocomportamental Pérez-Santos. Anuario de Psicología Clínica y de la Salud, (7), 51-50.

Muñiz, E (2014). Pensar el cuerpo de las mujeres: cuerpo, belleza y feminidad. Una necesaria mirada feminista. Revista Sociedade e Estado, 29(2), 425-432.

Navajas, N. (2017). La gordofobia es un problema del Trabajo Social. Revista Internacional de Trabajo Social y Bienestar, (6), 37-45.

Noronha, A. Deufel, C. (2014). Reflexões teóricas sobre a gordofobia na mídia: o corpo na contemporaneidade noronha. $V$ Seminario Nacional de Pesquisa em Educacao.

Nutter, S., Ireland, A., Alberga, A., Brun, I., Lefebvre, D., Hayden, A. \& Russell, S. (2019) Weight Bias in Educational Settings: a Systematic Review. Current Obesity Reports, (8), 185-200, DOI: https://doi.org/10.1007/s13679-019-00330-8

OAC. (2018). Comprensión del Estigma de la Obesidad. Recuperado de https://www.obesityaction.org/get-educated/publicresources/brochuresguides/comprension-del-estigma-dela-obesidad/

OMS (20 de julio de 2020). Sobrepeso y Obesidad. Recuperado de https://www.who.int/es/news-room/fact-sheets/detail/ obesity-and-overweight\#: :text=En\%20el\%20caso\%20 de\%20los,igual\%20o\%20superior\%20a\%2030.

O' Dea, J. A. (2005). Prevention of child obesity: 'First do no harm'. Health Education Research, (20), 259-265.

Palmeira, L., Cunha, M. \& Pinto-Gouveia, J. (2018). The weight of weight self-stigma in unhealthy eating behaviours: the mediator role of weight-related experiential avoidance. $E a$ ting and Weight Disorders - Studies on Anorexia, Bulimia and Obesity, 23(6), 785-796. DOI: https://doi.org/10.1007/ s40519-018-0540-Z 
Petermann, F., Martinez, M., Villagran, M., Ulloa, N., Nazar, G., Troncoso, C., Garrido, A., Mardones, L., Lanuza, F., Leiva, AM., Lasserre, N., Martorell, M. y Celis, C. (2020). Desde una mirada global al contexto chileno: ¿Qué factores han repercutido en el desarrollo de obesidad en Chile? (Parte 1). Revista Chilena de Nutrición, 47(2), 299-306. DOI: http://dx.doi.org/10.4067/ S0717-75182020000200299

Plá, I., Adam, A. y Bernabeu, I. (2013). Estereotipos y prejuicios de género: factores determinantes en Salud Mental. Norte de salud mental, 11(46), 20-28.

Puhl, R. \& Heuer, C. (2010). The Stigma of Obesity: A Review and Update. American Journal of Public Health, 17(5), 941-64.

Puhl, R. M. \& Latner, J. D. (2007). Stigma, obesity, and the health of the nation's children. Psychological Bulletin, 133(4), 557-580.

Puhl, R. \& Brownell KD. (2003) Ways of coping with obesity stigma: conceptual review and analysis. Eat Behav, 4(1), 53-78.

Puhl, R., Latner, JD., Brien, K., Luediche, J., Danielsdottir, S. \& Forhan, M. (2015). A multinational examination of weight bias: predictors of anti-fat attitudes across four countries. International Journal of Obesity, (39), 1166-1173.

Robinson, B., Bacon, L. C. \& O’Reilly, J. (1993). Fat phobia: Measuring, understanding, and changing anti-fat attitudes. International Journal of Eating Disorders, 14(4), 467-480.

Russell, S. \& Grace, A. (2015). A call for social justice and best practices for the integrated prevention of eating disorders and obesity. Eat Disord, (24), 54-62. DOI: https://doi. org/10.1080/10640266. 2015.1113829

Saavedra, J. y Robles, Y. (2011). La discriminación y su influencia en la Salud Mental. Anales de Salud Mental, (27), 9-13.

Strings, S. (2019). Fearing the Black Body: The Racial Origins of Fat Phobia. New York University Press.

Suárez, C. (2017). Gordofobia: Un tránsito entre la enfermedad y la cosificación del cuerpo femenino. (Trabajo de Grado). Universidad La Laguna.

Sykes, H. \& McPhail, D. (2008). Unbearable Lessons: Contesting Fat Phobia in Physical Education. Sociology of Sport Journal, (25), 66-96.

Thuan, J. F. \& Avignon, A (2005). Obesity Management: Attitudes and Practices of French General Practitioners in a Region of 
France. International Journal of Obesity, 29(9), 1100-1106. DOI: 10.1038/sj.ijo.0803016.

Washington, R. (2011). Childhood obesity: issues of weight bias. Preventing Chronic Diseases, 8(5), 4-94.

Wright, E., Gronfein, W. \& Owens, T. (2000). Deinstitutionalization, social rejection, and the Self-esteem of former mental patiens. Journal of Health and Social Behavior, 41(1), 68-90. 